\title{
Development and automation of a dietary feedback system for the delivery of personalised dietary advice
}

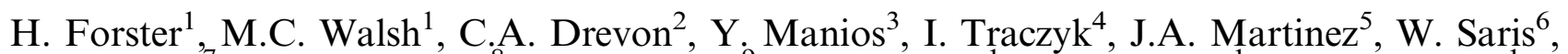 \\ H. Daniel ${ }^{7}$, J.A. Lovegrove ${ }^{8}$, J.C. Mathers ${ }^{9}$, M.J. Gibney ${ }^{1}$, E.R. Gibney ${ }^{1}$ and L. Brennan ${ }^{1}$, \\ on behalf of the Food4Me study \\ ${ }^{1}$ UCD Institute of Food and Health, University College Dublin, Ireland, ${ }^{2}$ Department of Nutrition, University of Oslo, \\ Norway, ${ }^{3}$ Department of Nutrition and Dietetics, Harokopio University, Athens, Greece, ${ }^{4}$ National Food \& Nutrition \\ Institute, Poland, ${ }^{5}$ Department of Nutrition, Food Science and Physiology, University of Navarra, Spain, ${ }^{6}$ Department \\ of Human Biology, NUTRIM, Maastricht University, The Netherlands, ${ }^{7}$ ZIEL Research Center of Nutrition and Food \\ Sciences, Technische Universität München, Germany, ${ }^{8}$ Hugh Sinclair Unit of Human Nutrition and Institute for \\ Cardiovascular and Metabolic Research, University of Reading, RG6 6AP, UK and ${ }^{9}$ Human Nutrition Research \\ Centre, Newcastle University, NE4 5PL, UK
}

This abstract was awarded a poster presentation prize sponsored by Sugar Nutrition UK and the Food Safety Authority of Ireland.

Diets high in saturated fat and salt and low in fibre, fruit and vegetables, have long been associated with increased risk of noncommunicable diseases $^{(1)}$. While existing public health promotion policies across Europe aim to promote healthy eating, the prevalence of undesirable diets is still high ${ }^{(2)}$. The objectives of the present study were to (i) describe the development of a feedback system for the delivery of personalised dietary advice and (ii) examine the use and automation of this feedback system within the Food4Me Proof-of-Principle (PoP) study.

The development of the feedback system consisted of 4 components: development of a system for categorising nutritional intakes; development of a method for prioritising 3 nutrient goals for subsequent dietary advice; designing decision trees to link nutritional intakes to feedback messages and the development of feedback reports. The system was used manually by researchers to provide personalised nutrition advice during the Food4Me study ${ }^{(3)}$. Following completion of the study the system was automated (Creme Global) and the level of agreement between the manual and automated systems was compared.

In total, 16 dietary decision trees were developed linking nutrient intakes to an archive of 92 feedback messages, (example in Fig. 1). All feedback messages were developed to consist of practical food-based dietary goals and tips to help improve nutrient intake.

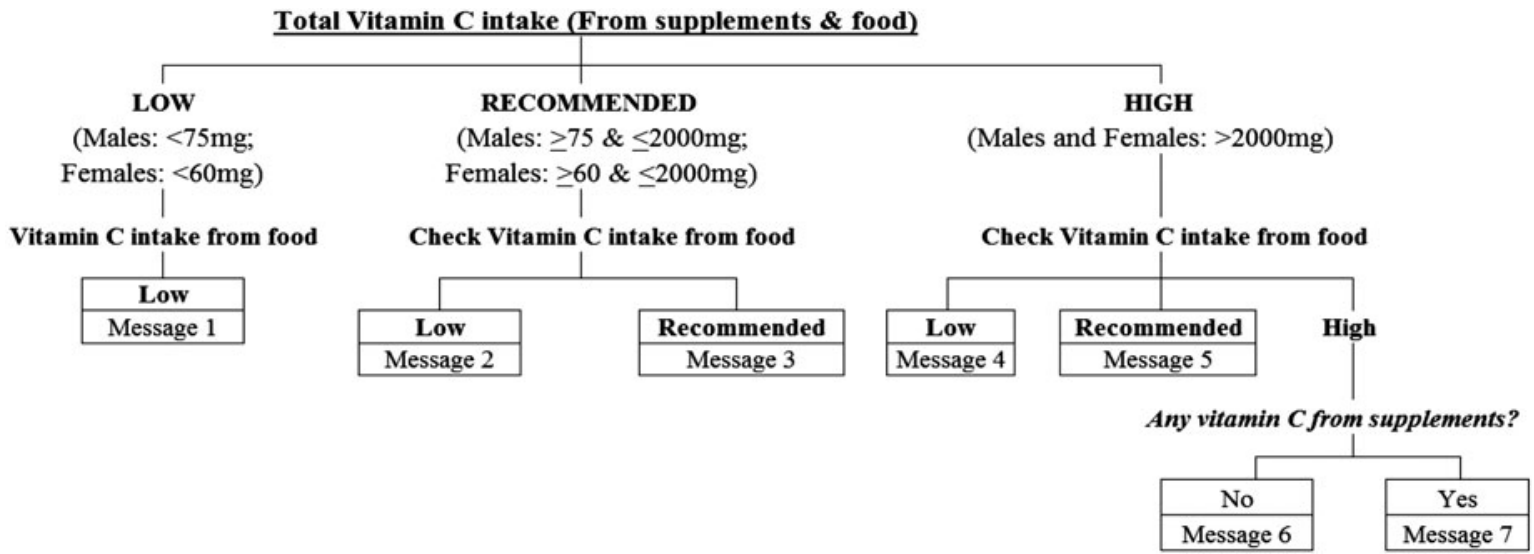

Fig. 1. Vitamin $\mathrm{C}$ decision tree.

A total of 369 participants across 7 centres, randomised to receive personalised nutrition advice based on dietary assessment, were included in the analysis. Saturated fat, salt and dietary fibre were most frequently targeted nutrient-related goals provided to $72 \%$, $72 \%$ and $39 \%$ of participants, respectively. Overall agreement between the manual and automated systems, in selecting the top 3 nutrient-related goals for personalised dietary advice, was highest for goals 1 and 2 and lower for goal 3, averaging at $91 \%$, $87 \%$ and $63 \%$, respectively.

The dietary feedback system was a comprehensive method for delivering consistent personalised dietary advice. The good agreement between the manual and automated systems suggests that automated systems can be developed successfully to provide personalised dietary advice as components of Connected Health systems.

This work was funded by Food4Me (KBBE.2010·2·3-02, Project no. 265494)

1. Vandevijvere S \& Swinburn B (2014) BMJ Open 4, e005339.

2. Capacci S, Mazzocchi M, Shankar B et al. (2012) Nutr Rev 70, 188-200.

3. Celis-Morales C, Livingstone KM, Marsaux CFM et al. (2015) Genes Nutr 10, 450. 